maximal firing in a topographical map. But Brand et al. also found that inhibitory inputs, which are not involved in the traditional model, were crucial for generating the ITD sensitivity of the MSO neurons. When inhibitory transmission was blocked, the MSO neurons all fired maximally at an ITD of around $0 \mu \mathrm{s}$. The authors conclude that precisely timed inhibition is needed to generate the range of ITD sensitivities observed in the MSO.

It remains to be seen whether this mechanism is common to all mammals, or whether it is specific to those, like gerbils, that have very small heads and therefore experience very small ITDs. But it is clear that there is more to sound localization than meets the eye or ear.

Rachel Jones

(4) References and links ORIGINAL RESEARCH PAPER Brand, A. et al. Precise inhibition is essential for microsecond interaural time difference coding. Nature $\mathbf{4 1 7}$ interaural time dist $543-547$ (2002) 543-547 (2002)
FURTHER READING Pollack, G. D. Model hearing. Nature 417, 502-503 (2002)

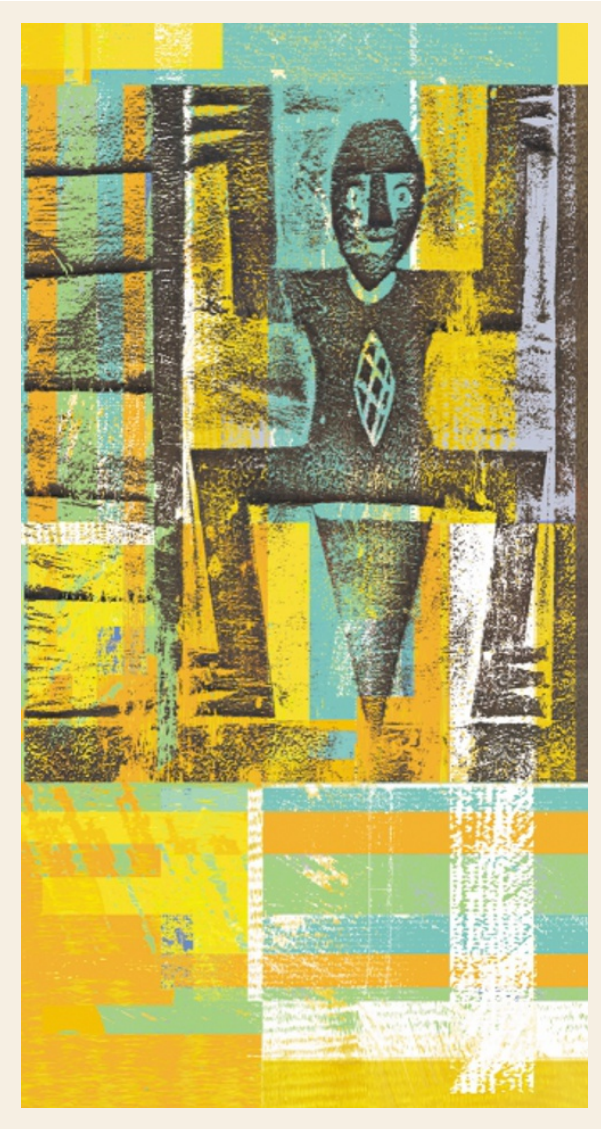

\section{PSYCHIATRIC DISORDERS}

\section{On an even keel}

A common mode of action of three structurally diverse mood-stabilizing drugs has been identified for the first time. In a paper published in Nature, two research groups led by Mudge and Harwood show that lithium, carbamazepine (CBZ) and valproic acid (VPA) have similar effects on the dynamic properties of sensory neuron growth cones. Their findings implicate inositol depletion in the mechanism of action of these drugs, and provide clues to the molecular basis of bipolar affective disorder (manic depression).

Lithium is one of the most effective drugs for the treatment of bipolar disorder. The inositoldepletion hypothesis, which hinges on the ability of lithium to inhibit inositol monophosphatase and inositol polyphosphatase, has guided research on the therapeutic actions of this drug for more than a decade. However, there is evidence to suggest that lithium might exert its effects by inhibiting glycogen synthase kinase 3 (GSK3). Inositol depletion has also been implicated in the therapeutic actions of VPA, although some evidence points to histone deacetylase (HDAC) as the target of this treatment. The search for the mechanism of action of mood-stabilizing drugs has, to some extent, been hampered by a lack of knowledge of the neurobiology of mood disorders. Mudge and Harwood reasoned that if a shared mechanism of action could be identified for three of the most commonly used mood stabilizers - lithium, CBZ and VPA - then the molecular targets that are central to their effects, and perhaps the underlying abnormality in bipolar disorder, might be uncovered.

In cultured explants of sensory neurons from newborn rat dorsal root ganglia, Mudge and colleagues showed that lithium, CBZ and VPA had differential effects on the structural features of sensory neuron axons. However, when cultures were viewed by time-lapse video microscopy, all three drugs were found to have a similar effect on the dynamic behaviour of sensory neuron growth cones - to reduce their frequency of collapse. Moreover, growth cones were enlarged after treatment with each of these drugs, possibly reflecting the inhibition of growth cone collapse. These effects were abolished by the addition of inositol, implicating inositol phosphate signalling in this common response; by contrast, the shared actions of these drugs did not seem to depend on GSK3 or HDAC.

Harwood and colleagues examined the effects of lithium and VPA on the development of Dictyostelium. During early development, cells aggregate by chemotaxis, and this process is disrupted when inositol-1,4,5-trisphosphate

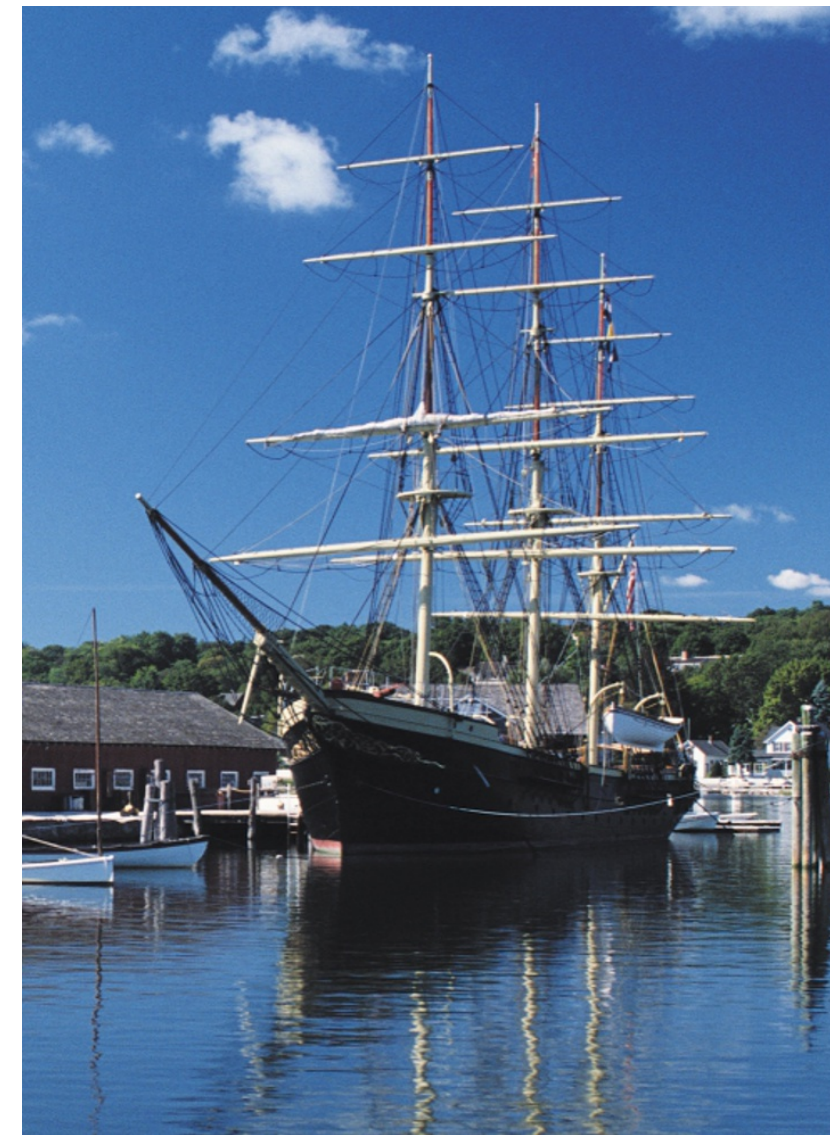

(Ins $\left.(1,4,5) \mathrm{P}_{3}\right)$ signalling is suppressed by lithium. They showed that inhibition of Dictyostelium aggregation by VPA also seems to depend on Ins $(1,4,5) \mathrm{P}_{3}$ signalling, and that resistance to both lithium and VPA can be afforded by deletion of the gene that codes for prolyl oligopeptidase, a cytoplasmic protein that can cleave short oligopeptides at prolyl bonds. In view of this finding, the authors looked for a link between prolyl oligopeptidase and the shared actions of the mood-stabilizing drugs on neurons. They found that two specific inhibitors of prolyl oligopeptidase could abolish the effects of lithium, CBZ and VPA on growth cone collapse and spread area.

Although the physiological function of prolyl oligopeptidase is unknown, these data indicate that it might be involved in the regulation of inositol metabolism in mammalian cells. Intriguingly, plasma concentrations of the human homologue of this protein are reported to be elevated in mania and reduced in depression; however, whether abnormalities in prolyl oligopeptidase activity are central to the pathophysiology of bipolar affective disorder remains to be seen. We may be some way from attaining a full description of the neurobiology of bipolar disorder, but this study provides a clear focus for future studies of its aetiology and treatment.

Rebecca Craven

(1) References and links

ORIGINAL RESEARCH PAPER Williams, R. S. B. et al. A common

mechanism of action for three mood-stabilizing drugs. Nature $\mathbf{4 1 7}$, 292-295 (2002)

WEB SITES

Anne Mudge's lab: http://www.ucl.ac.uk/LMCB/pages/mudge.html Encyclopedia of Life Sciences: http://www.els.net/ mood disorders 